\title{
Solid-Phase Synthesis of Anandamide Analogues
}

\author{
Longwu Qi, Michael M. Meijler, Sang-Hyeup Lee, Chengzao Sun and Kim D. Janda* \\ Department of Chemistry, The Scripps Research Institute and The Skaggs Institute for Chemical Biology, \\ 10550 North Torrey Pines Road, La Jolla, California 92037
}

kdjanda@scripps.edu

\section{Supporting Information}

General Methods. Unless otherwise noted, materials were obtained from commercial suppliers and were used without further purification. THF was distilled from sodiumbenzophenone. The ion-exchange resin used in product purification, Dowex 50WX2-200, was washed with distilled water, $\mathrm{MeOH}$, and $\mathrm{CH}_{2} \mathrm{Cl}_{2}$ prior to use. The filtration/workup cartridges were prepared by placing $\sim 1 \mathrm{~cm}^{3}$ of Florisil and $\sim 2 \mathrm{~cm}^{3}$ of the resin in a 5-mL plastic syringe equipped with a polyethylene frit. All of the glassware used in the solidphase synthesis was silanized by treating with sigmacote. Flash chromatography was carried out using Merck silica gel 60 (230-400 mesh). Preparative TLC was carried out using Merck $60 \mathrm{~F}_{254}$ plates $(0.5$ or $1.0 \mathrm{~mm})$ with ethyl acetate/hexanes or methanol/methylene chloride mixtures as eluants.

FT-IR spectra were recorded using a Thermo Nicolet AVATA 360 spectrometer equipped with a golden gate single reflection diamond ATR accessory. NMR spectra were recorded using either a Bruker DRX-500 or 600 spectrometer and calibrated using residual undeuterated solvent as an internal reference. High-resolution mass spectra (HRMS) were recorded at The Scripps Research Institute using MALDI-FTMS techniques. LC-MS spectra were obtained on a HP-1100, using a flow rate of 0.75 $\mathrm{mL} / \mathrm{min}$ in a gradient of $25-99 \%$ acetonitrile in water ( $0.5 \%$ formic acid) in $8-12 \mathrm{~min}$.

Final products were fully characterized $\left({ }^{1} \mathrm{H}-,{ }^{13} \mathrm{C}-\mathrm{NMR}\right.$ and MS), except for a few compounds that did not yield sufficient material for reliable ${ }^{13} \mathrm{C}-\mathrm{NMR}$ data. For compound $4 \mathrm{i}$ also the resolution of the ${ }^{1} \mathrm{H}-\mathrm{NMR}$ spectrum was insufficient for publication, however, a clean mass spectrum was obtained. Intermediates were characterized by ${ }^{1} \mathrm{H}-\mathrm{NMR}$, and ${ }^{13} \mathrm{C}-\mathrm{NMR}$ or $\mathrm{MS}$.

General method for resin washing and drying: the resin was washed with DMF (3x), $\mathrm{H}_{2} \mathrm{O} / \mathrm{DMF}(1: 1)(3 \mathrm{x})$, methanol (3x), methylene chloride (3x) and the washing cycle was repeated three times. The resin was dried in vacuo overnight.

Off-beads analysis: about $50 \mathrm{mg}$ resin was treated by $50 \%$ TFA in methylene chloride, shaken for $30 \mathrm{~min}$, followed by filtration and washed with methylene chloride (3x); the solvent was removed and the residue was taken up in $\mathrm{CH}_{2} \mathrm{Cl}_{2}$ and concentrated (2x) and dried in vacuo. The sample was weighed and analyzed by NMR.

General method for the $\mathrm{Cu}$-mediated coupling reactions in solid phase: 
The terminal alkyne and propargyl bromide or chloride, $\mathrm{CuI}, \mathrm{NaI}, \mathrm{K}_{2} \mathrm{CO}_{3}$ were mixed in DMF and the mixture was shaken for 3 days. The reaction was monitored either by IR or by off-beads NMR analysis. The yield and purity were determined by off-beads analysis.

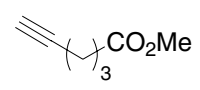

7 Ester 7: A stirred solution of 5-hexynoic acid (5 g, $44.6 \mathrm{mmol}), \mathrm{p}$-TSA (monohydrate) $(58 \mathrm{mg}, 0.3 \mathrm{mmol})$ in $\mathrm{MeOH}(8 \mathrm{~mL})$ and $\mathrm{CH}_{2} \mathrm{Cl}_{2}(17 \mathrm{~mL})$ was refluxed for $24 \mathrm{~h}$. The mixture was quenched with saturated $\mathrm{NaHCO}_{3}$ and the organic layer was separated. The aqueous phase was extracted with $\mathrm{CH}_{2} \mathrm{Cl}_{2}(3 \times 10 \mathrm{~mL})$. The combined organic layers were dried over $\mathrm{MgSO}_{4}$ and the solvent was removed in vacuo after filtration. $4.2 \mathrm{~g}(33.4 \mathrm{mmol})$ of 7 was obtained $(75 \%$ yield $) .{ }^{1} \mathrm{H}$ NMR $\left(\mathrm{CDCl}_{3}, 500\right.$ $\mathrm{MHz}): \delta 3.70(\mathrm{~s}, 3 \mathrm{H}), 2.50(\mathrm{t}, J=7.2 \mathrm{~Hz}, 2 \mathrm{H}), 2.30(\mathrm{dt}, J=7.2,2.8 \mathrm{~Hz}, 2 \mathrm{H}), 2.0(\mathrm{t}, J=2.8$ $\mathrm{Hz}, 1 \mathrm{H}$ ), 1.88 (quint, $J=7.2 \mathrm{~Hz}, 2 \mathrm{H}$ ).

$\mathrm{Cl}$

$\overline{8}$ OH Alcohol 8: To a solution of but-2-yn-1,4-diol $(86.0 \mathrm{~g}, 1 \mathrm{~mol})$ and pyridine $(89.0 \mathrm{~mL}, 1.1 \mathrm{~mol})$ in benzene $(100 \mathrm{~mL})$ was added thionyl chloride $(80.2 \mathrm{~mL}, 1.1 \mathrm{~mol})$ dropwise over a period of $6 \mathrm{~h}$, while the temperature was maintained between $10{ }^{\circ} \mathrm{C}$ to 20 ${ }^{\circ} \mathrm{C}$. The reaction mixture was then stirred overnight at room temperature. The mixture was poured into ice water $(250 \mathrm{~mL})$ and the benzene layer was separated. The aqueous phase was extracted with ether $(4 \times 100 \mathrm{~mL})$ and the combined organic layers were washed with saturated $\mathrm{NaHCO}_{3}$, followed by water and brine. The organic phase was dried over $\mathrm{MgSO}_{4}$ and the solvent was removed in vacuo. Purification of the residue oil by distillation $\left(55^{\circ} \mathrm{C} / 0.7 \mathrm{~mm} \mathrm{Hg}\right)$ provided $52.3 \mathrm{~g}$ of 8 as colorless oil in $50 \%$ yield. ${ }^{1} \mathrm{H}$ NMR $\left(\mathrm{CDCl}_{3}, 500 \mathrm{MHz}\right): \delta 4.33(\mathrm{dt}, J=6.3,1.9 \mathrm{~Hz}, 2 \mathrm{H}), 4.18(\mathrm{t}, J=1.9 \mathrm{~Hz}, 2 \mathrm{H})$.

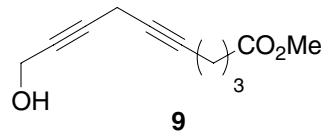

Diyne 9: A mixture of $\mathrm{K}_{2} \mathrm{CO}_{3}(4.64 \mathrm{~g}, 33.6 \mathrm{mmol}), \mathrm{CuI}(12.80 \mathrm{~g}, 33.6$ mmol), NaI (5.04 g, $33.6 \mathrm{mmol})$, ester 7 (4.23 g, $33.6 \mathrm{mmol})$ and alcohol 8 (3.53 g, 33.6 mmol) in DMF $(68 \mathrm{~mL})$ was stirred overnight at $25{ }^{\circ} \mathrm{C}$. The mixture was diluted with EtOAc $(100 \mathrm{~mL})$ and plugged through a pad of celite. It was washed with saturated $\mathrm{NH}_{4} \mathrm{Cl}\left(4 \times 20 \mathrm{~mL}\right.$ ) and followed by brine. The solution was dried over $\mathrm{MgSO}_{4}$ and the solvent was removed in vacuo after filtration. The residue was purified by flash chromatography on silica gel (EtOAc/Hex: 2/3) to give $6.06 \mathrm{~g}(93 \%)$ of 9 as yellow oil. ${ }^{1} \mathrm{H} \mathrm{NMR}\left(\mathrm{CDCl}_{3}, 500 \mathrm{MHz}\right): \delta 4.27(\mathrm{dt}, J=6.0,2.2 \mathrm{~Hz}, 2 \mathrm{H}), 3.70$ (s, 3H), 3.20 (quint, $J=2.2 \mathrm{~Hz}, 2 \mathrm{H}$ ), 2.48 (t, $J=7.4 \mathrm{~Hz}, 2 \mathrm{H}$ ), 2.27 (tt, $J=6.7,2.2 \mathrm{~Hz}, 2 \mathrm{H}$ ), 1.87 (quint, $J=6.7 \mathrm{~Hz}$, $2 \mathrm{H})$.

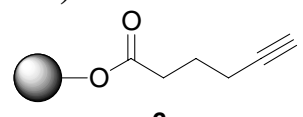

$6 \quad$ Polymer supported 5-hexynoic acid (6): A solution of 5-hexynoic acid (0.434 mL, $3.93 \mathrm{mmol})$ in $15 \mathrm{~mL}$ of DMF was treated with DIC (0.616 mL, $3.93 \mathrm{mmol})$ and stirred for $15 \mathrm{~min}$ at $\mathrm{rt}$. Wang resin $(1.36 \mathrm{~g}, 0.96 \mathrm{mmol} / \mathrm{g}, 1.31 \mathrm{mmol})$ was then added followed by DMAP $(48.0 \mathrm{mg}, 0.39 \mathrm{mmol})$ and $\mathrm{NMM}(0.45 \mathrm{~mL}, 3.93 \mathrm{mmol})$. The suspension was stirred overnight at $\mathrm{rt}$ under Ar. The resin was washed by the general washing method and dried. $66.5 \mathrm{mg}$ of the dry resin was cleaved with $50 \%$ TFA in 
$\mathrm{CH}_{2} \mathrm{Cl}_{2}$ according to the general method to give $10 \mathrm{mg}$ of acid with a loading value of $1.34 \mathrm{mmol} / \mathrm{g}$ (theoretical loading of the resin $0.877 \mathrm{mmol} / \mathrm{g}$, quantitative).

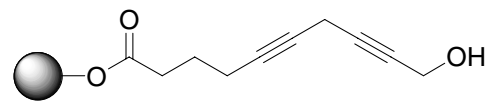

10a: To a mixture of $\mathrm{CuI}(45.7 \mathrm{mg}, 0.24 \mathrm{mmol}), \mathrm{NaI}(36.0$ $\mathrm{mg}, 0.24 \mathrm{mmol})$ and $\mathrm{K}_{2} \mathrm{CO}_{3}(33.1 \mathrm{mg}, 0.24 \mathrm{mmol})$ in DMF $(0.6 \mathrm{~mL})$ was added resin 6 $(59.9 \mathrm{mg}, 1.34 \mathrm{mmol} / \mathrm{g}, 0.08 \mathrm{mmol})$ followed by alcohol 8 (84.3 mg, $0.8 \mathrm{mmol})$ under Ar. The suspension was shaken for 3 days at rt. The resin was filtered and washed according to the general method. $21.2 \mathrm{mg}$ of the resin was cleaved by the TFA method to give 4.6 $\mathrm{mg}$ solid with a loading value of $1.22 \mathrm{mmol} / \mathrm{g}$ (theoretical: $1.25 \mathrm{mmol} / \mathrm{g}$, yield $97 \%, 92$ $\%$ purity from ${ }^{1} \mathrm{H}$ NMR). For the corresponding acid obtained from the resin by TFA, ${ }^{1} \mathrm{H}$ NMR ( $\left.\mathrm{CDCl}_{3}, 400 \mathrm{MHz}\right): \delta 4.27(\mathrm{t}, J=2.2 \mathrm{~Hz}, 2 \mathrm{H}), 3.17$ (quint., $\left.J=2.3 \mathrm{~Hz}, 2 \mathrm{H}\right), 2.49$ (t, $J=7.2 \mathrm{~Hz}, 2 \mathrm{H}), 2.27$ (m, 2H), 1.84 (quint., $J=7.1 \mathrm{~Hz}, 2 \mathrm{H}), 1.25(-\mathrm{OH}) .{ }^{13} \mathrm{C}$ NMR (500 $\left.\mathrm{MHz}, \mathrm{CDCl}_{3}\right): \delta 178.33,80.65,79.59,78.37,74.66,51.20,32.70,23.35,18.13,9.82$.

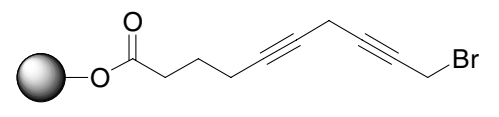

10b: Resin 10a (116.1 mg, $0.146 \mathrm{mmol})$ was swollen in $\mathrm{DCM}(1.7 \mathrm{~mL})$ for $15 \mathrm{~min}$, and then cooled to $-40{ }^{\circ} \mathrm{C}$ under $\mathrm{Ar}$. $\mathrm{CBr}_{4}(96.0 \mathrm{mg}, 0.28$ mmol) was added to the suspension followed by a solution of $\mathrm{Ph}_{3} \mathrm{P}(77.0 \mathrm{mg}, 0.28 \mathrm{mmol})$ in DCM $(0.5 \mathrm{~mL})$. The suspension was stirred mildly at $-20{ }^{\circ} \mathrm{C}$ for $1 \mathrm{~h}$. The suspension was diluted with DCM (2 mL), filtered and washed with DCM (5 x $15 \mathrm{~mL})$. The resin was dried in vacuo overnight. Off-bead analysis: $34.6 \mathrm{mg}$ of resin gave $6.5 \mathrm{mg}$ of solid:loading value $0.78 \mathrm{mmol} / \mathrm{g}$ (theoretical: $1.1 \mathrm{mmol} / \mathrm{g}$, yield $71 \%$ ), purity $88 \% .{ }^{1} \mathrm{H}$ NMR $\left(\mathrm{CDCl}_{3}, 500 \mathrm{MHz}\right): \delta 3.92(\mathrm{t}, J=2.4 \mathrm{~Hz}, 2 \mathrm{H}), 3.22$ (quint, $\left.J=2.3 \mathrm{~Hz}, 2 \mathrm{H}\right), 2.50(\mathrm{t}$, $J=7.4 \mathrm{~Hz}, 2 \mathrm{H}), 2.28$ (m, 2H), 1.84 (quint., $J=7.2 \mathrm{~Hz}, 2 \mathrm{H}) .{ }^{13} \mathrm{C} \mathrm{NMR}\left(500 \mathrm{MHz}, \mathrm{CDCl}_{3}\right)$ : $\delta 178.41,81.75,79.79,75.38,74.05,69.29,32.56,23.44,18.03,14.78,10.05$.

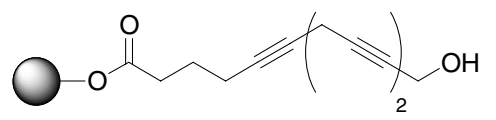

11a: To a mixture of $\mathrm{CuI}(371.3 \mathrm{mg}, 1.95 \mathrm{mmol})$, NaI (292.6 mg, $1.95 \mathrm{mmol}$ ) and $\mathrm{K}_{2} \mathrm{CO}_{3}(269.1 \mathrm{mg}, 1.95 \mathrm{mmol})$ in DMF (3 mL) was added resin $10 \mathrm{a}(304.6 \mathrm{mg}, 0.78 \mathrm{mmol} / \mathrm{g}, 0.24 \mathrm{mmol})$ followed by propargyl alcohol $(142.0 \mu \mathrm{L}$, $2.43 \mathrm{mmol})$ at $\mathrm{rt}$ under Ar. The vial was capped firmly and shaken for 3 days. After filtration and washing by the general method, the resin was dried in vacuo. Off-bead analysis: $34.9 \mathrm{mg}$ resin gave $5.1 \mathrm{mg}$ of solid compound:loading value $0.70 \mathrm{mmol} / \mathrm{g}$ (theoretical: $0.81 \mathrm{mmol} / \mathrm{g}$, yield $86 \%$ ), purity $85 \%$. ${ }^{1} \mathrm{H}$ NMR $\left(\mathrm{CDCl}_{3}, 500 \mathrm{MHz}\right): \delta 4.27$ $(\mathrm{t}, J=2.1 \mathrm{~Hz}, 2 \mathrm{H}), 3.20(\mathrm{t}, J=2.3 \mathrm{~Hz} 2 \mathrm{H}), 3.13(\mathrm{t}, J=2.3 \mathrm{~Hz}, 2 \mathrm{H}), 2.50(\mathrm{t}, J=7.5 \mathrm{~Hz}, 2 \mathrm{H})$, $2.27(\mathrm{~m}, 2 \mathrm{H}), 1.83(\mathrm{~m}, 2 \mathrm{H}) .{ }^{13} \mathrm{C} \mathrm{NMR}\left(500 \mathrm{MHz}, \mathrm{CDCl}_{3}\right): \delta 178.03,80.09,79.39,78.64$, 75.27, 74.92, 73.83, 51.21, 32.48, 29.68, 23.43, 18.02, 9.86 .

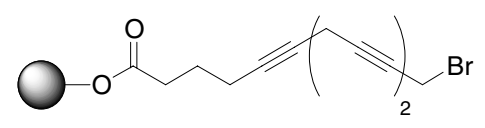

11b: resin 11a was treated by the same method used to prepare 10b: 11a $(92.2 \mathrm{mg}, 0.70 \mathrm{mmol} / \mathrm{g}, 0.064 \mathrm{mmol}), \mathrm{CBr}_{4}(31.6 \mathrm{mg}, 0.095 \mathrm{mmol})$ and $\mathrm{Ph}_{3} \mathrm{P}(25.0 \mathrm{mg}, 0.095 \mathrm{mmol})$ in DCM $(1.5 \mathrm{~mL})$. Off-bead analysis: $40.0 \mathrm{mg}$ of the resin gave $7.3 \mathrm{mg}$ of solid compound: loading value $0.65 \mathrm{mmol} / \mathrm{g}$ (theoretical: $0.66 \mathrm{mmol} / \mathrm{g}$, yield $98 \%$ ), purity $83 \% .{ }^{1} \mathrm{H} \mathrm{NMR}\left(\mathrm{CDCl}_{3}, 500 \mathrm{MHz}\right): \delta 3.91(\mathrm{t}, J=2.3 \mathrm{~Hz}, 2 \mathrm{H}), 3.23(\mathrm{t}$, 
$J=2.3 \mathrm{~Hz}, 2 \mathrm{H}), 3.13(\mathrm{t}, J=2.4 \mathrm{~Hz}, 2 \mathrm{H}), 2.49(\mathrm{t}, J=7.4 \mathrm{~Hz}, 2 \mathrm{H}), 2.26(\mathrm{~m}, 2 \mathrm{H}), 1.82$ (quint, $J=7.2 \mathrm{~Hz}, 2 \mathrm{H}) .{ }^{13} \mathrm{C} \mathrm{NMR}\left(500 \mathrm{MHz}, \mathrm{CDCl}_{3}\right): \delta 178.82,81.67,79.85,77.69,75.89,75.18$, $73.78,69.73,32.98,23.92,18.48,18.15,10.54$.

Compounds 5: prepared from resin $\mathbf{1 1 b}$ by the same condition as used to prepare 11a: resin 11b (1 eq), and alkyne (10 eq) were added to the mixture of $\mathrm{CuI}(8 \mathrm{eq}), \mathrm{NaI}(8 \mathrm{eq})$, and $\mathrm{K}_{2} \mathrm{CO}_{3}(8 \mathrm{eq})$ in DMF (9 mL/ $\mathrm{g}$ resin) and shaken for 3 days. After filtration and washing by the general method, the resin was dried in vacuo.

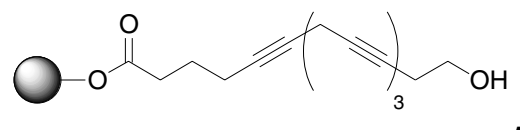

5a 5a: off-bead analysis: $22.5 \mathrm{mg}$ of the resin gave $2.7 \mathrm{mg}$ of compound:, loading value $0.44 \mathrm{mmol} / \mathrm{g}$ (theoretical: $0.65 \mathrm{mmol} / \mathrm{g}$, yield $68 \%$ ), purity $70 \%$. For the corresponding acid obtained from the resin by TFA, ${ }^{1} \mathrm{H}$ NMR $\left(\mathrm{CDCl}_{3}, 500\right.$ $\mathrm{MHz}): \delta 3.75(\mathrm{t}, J=6.2 \mathrm{~Hz}, 2 \mathrm{H}), 3.14(\mathrm{~m}, 6 \mathrm{H}), 2.49(\mathrm{~m}, 2 \mathrm{H}), 2.45(\mathrm{~m}, 2 \mathrm{H}), 2.26(\mathrm{~m}, 2 \mathrm{H})$, 1.82 (quint, $J=7.1 \mathrm{0Hz}, 2 \mathrm{H}$ ).

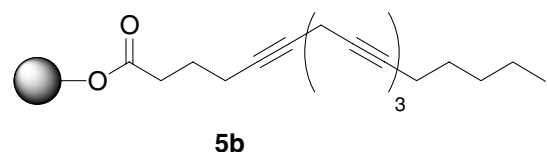

$5 \mathrm{~b}$

5b: off-bead analysis: $21.2 \mathrm{mg}$ of the resin gave $4.1 \mathrm{mg}$ of compound:, loading value $0.65 \mathrm{mmol} / \mathrm{g}$ (theoretical: $0.62 \mathrm{mmol} / \mathrm{g}$, quantitative), purity: $71 \%$. For the corresponding acid obtained from the resin by TFA, ${ }^{1} \mathrm{H}$ NMR $\left(\mathrm{CDCl}_{3}, 400 \mathrm{MHz}\right): \delta 3.14(\mathrm{~m}, 6 \mathrm{H}), 2.49(\mathrm{t}, J=7.43 \mathrm{~Hz}, 2 \mathrm{H}), 2.26(\mathrm{~m}, 2 \mathrm{H}), 2.15(\mathrm{~m}, 2 \mathrm{H})$, 1.82 (quint, $J=7.215 \mathrm{~Hz}, 2 \mathrm{H}), 1.49(\mathrm{~m}, 2 \mathrm{H}), 1.32(\mathrm{~m}, 4 \mathrm{H}), 0.89(\mathrm{t}, J=6.8 \mathrm{0Hz}, 3 \mathrm{H})$.

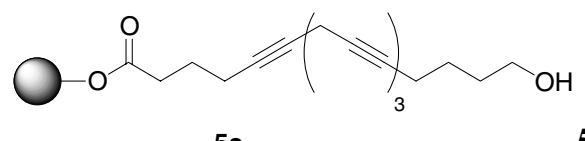

$5 c$

5c: off-bead analysis: $27.7 \mathrm{mg}$ of the resin gave $3.8 \mathrm{mg}$ of compound: loading value $0.46 \mathrm{mmol} / \mathrm{g}$ (theoretical: $0.59 \mathrm{mmol} / \mathrm{g}$, yield $78 \%$ ), purity $60 \%$. For the corresponding acid obtained from the resin by TFA, ${ }^{1} \mathrm{H}$ NMR $\left(\mathrm{CDCl}_{3}, 500\right.$ MHz): $\delta 4.38(\mathrm{t}, J=6.5 \mathrm{~Hz}, 2 \mathrm{H}), 3.14(\mathrm{~m}, 6 \mathrm{H}), 2.49(\mathrm{~m}, 2 \mathrm{H}), 2.26(\mathrm{~m}, 4 \mathrm{H}), 1.83(\mathrm{~m}, 4 \mathrm{H})$, $1.50(\mathrm{~m}, 2 \mathrm{H})$.

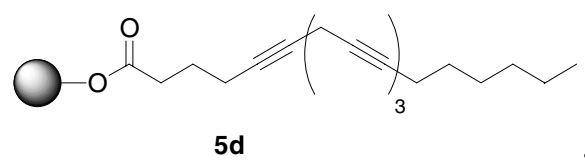

5d: off-bead analysis: $11.9 \mathrm{mg}$ of resin gave $2.9 \mathrm{mg}$ of compound: loading value $0.78 \mathrm{mmol} / \mathrm{g}$ (theoretical: $0.61 \mathrm{mmol} / \mathrm{g}$, quantitative, purity $65 \%$. For the corresponding acid obtained from the resin by TFA, ${ }^{1} \mathrm{H}$ NMR $\left(\mathrm{CDCl}_{3}, 400\right.$ $\mathrm{MHz}): \delta 3.13(\mathrm{~m}, 6 \mathrm{H}), 2.49(\mathrm{~m}, 2 \mathrm{H}), 2.26(\mathrm{~m}, 2 \mathrm{H}), 2.15(\mathrm{~m}, 2 \mathrm{H}), 1.82$ (quint. $J=7.4 \mathrm{~Hz}$, $2 \mathrm{H}), 1.48(\mathrm{~m}, 2 \mathrm{H}), 1.25(\mathrm{~m}, 7 \mathrm{H}), 0.89$ (t, $J=6.8 \mathrm{~Hz}, 3 \mathrm{H})$.

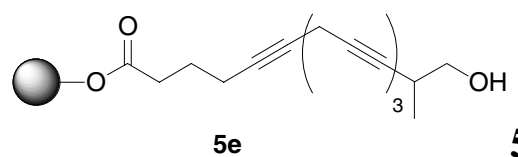

compound:, loading value $1.25 \mathrm{mmol} / \mathrm{g}$ (theoretical: $0.62 \mathrm{mmol} / \mathrm{g}$, quantitative, purity 
$33 \%$. For the corresponding acid obtained from the resin by TFA, ${ }^{1} \mathrm{H}$ NMR $\left(\mathrm{CDCl}_{3}, 400\right.$ MHz): $\delta 3.28(\mathrm{~m}, 2 \mathrm{H}), 3.13(\mathrm{~m}, 6 \mathrm{H}), 2.52(\mathrm{~m}, 3 \mathrm{H}), 2.28(\mathrm{~m}, 2 \mathrm{H}), 1.85(\mathrm{~m}, 2 \mathrm{H}), 1.25(\mathrm{~s}$, $1 \mathrm{H}), 0.88(\mathrm{t}, J=7.0 \mathrm{0Hz}, 3 \mathrm{H})$.

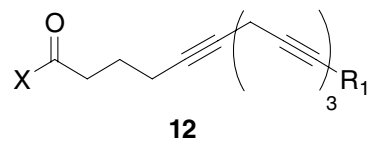

12: resin 5 was cleaved either by A) $50 \%$ TFA in DCM, $30 \mathrm{~min}, \mathrm{rt}$ as general method or $\mathrm{B}) \mathrm{Me}_{3} \mathrm{Al}$ (3eq)/amine (5eq), $0^{\circ} \mathrm{C} / 10$ min then overnight/rt; the mixture was quenched with water and stirred for $10 \mathrm{~min}$, then filtered through a filtration/workup cartridge to provide the crude product.<smiles>CC(C)=C(C)CCCC(=O)O</smiles>

12a: $144.0 \mathrm{mg}$ resin $\mathbf{5 a}$ was cleaved by $50 \%$ TFA in DCM, $1 \mathrm{~h}$, rt. $24.8 \mathrm{mg}$ of brown solid was obtained which was subjected to chromatography (silica gel, $\left.\mathrm{Et}_{2} \mathrm{O} / \mathrm{Hex}: 1 / 1\right)$ and two compounds were obtained: 12a (11.4 mg, 45\%) and 13a $(12 \mathrm{mg}, 45 \%) .{ }^{1} \mathrm{H}$ NMR $\left(\mathrm{CDCl}_{3}, 500 \mathrm{MHz}\right): \delta 3.70(\mathrm{t}, J=6.2 \mathrm{~Hz}, 2 \mathrm{H}), 3.15(\mathrm{dd}, J=2.4$ $\mathrm{Hz}, 12.5 \mathrm{~Hz}, 6 \mathrm{H}$ ), 2.50 (t, $J=7.4 \mathrm{~Hz}, 2 \mathrm{H}$ ), 2.25 (t, $J=6.9 \mathrm{~Hz}, 2 \mathrm{H}$ ), 1.83 (quint, $J=7.1 \mathrm{~Hz}$, 2H). HRMS (MALDI-FTMS) calcd. for $\mathrm{C}_{17} \mathrm{H}_{18} \mathrm{O}_{3}[\mathrm{M}+\mathrm{Na}]^{+} 293.1148$, found 293.1145.

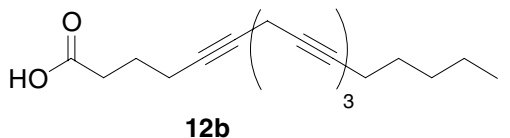

$12 b$

12b: resin 5b $(155.0 \mathrm{mg}, 0.09 \mathrm{mmol})$ was cleaved by method $\mathrm{A}$ as described above to give the crude product, which was purified by chromatography (silica gel, Et2O/Hex: 1/1) to provide pure product $\mathbf{1 2 b}$ as a white solid (21.3 mg, 80\%). ${ }^{1} \mathrm{H} \mathrm{NMR}\left(\mathrm{CDCl}_{3}, 500 \mathrm{MHz}\right): \delta 3.15(\mathrm{~m}, 6 \mathrm{H}), 2.45(\mathrm{t}, J=7.4 \mathrm{~Hz}, 2 \mathrm{H})$, $2.26(\mathrm{~m}, 2 \mathrm{H}), 2.15(\mathrm{~m}, 2 \mathrm{H}), 1.82$ (quint $J=7.1 \mathrm{~Hz}, 2 \mathrm{H}), 1.49$ (quint, $J=7.2 \mathrm{~Hz}, 2 \mathrm{H}$ ), 1.32 (m, 4H), 0.89 (t, $J=7.9 \mathrm{~Hz}, 3 \mathrm{H}) .{ }^{13} \mathrm{C}$ NMR $\left(500 \mathrm{MHz}, \mathrm{CDCl}_{3}\right): \delta$ 178.68, 80.96, 79.27, $75.24,74.92,74.88,74.29,74.03,73.57,32.58,31.06,29.68,28.39,23.48,22.19,18.66$, $18.06,13.97,9.82,9.75,9.72$.

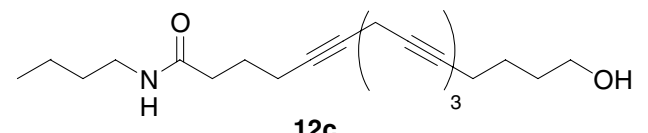

12c: resin 5c (106.2 mg, $0.049 \mathrm{mmol})$ was cleaved by method B to give product 12c $(18 \mathrm{mg}, 96 \%)$, purity $57 \%$. ${ }^{1} \mathrm{H}$ NMR $\left(\mathrm{CDCl}_{3}, 400\right.$ MHz): $\delta 3.56(\mathrm{t}, J=6.3 \mathrm{~Hz}, 2 \mathrm{H}), 3.24(\mathrm{~m}, 2 \mathrm{H}), 3.12(\mathrm{~m}, 6 \mathrm{H}), 2.28(\mathrm{~m}, 2 \mathrm{H}), 1.83(\mathrm{~m}, 4 \mathrm{H})$, $1.48(\mathrm{~m}, 4 \mathrm{H}), 1.35(\mathrm{~m}, 6 \mathrm{H}), 0.95(\mathrm{t}, J=7.5 \mathrm{~Hz}, 3 \mathrm{H}) . \mathrm{LC} / \mathrm{MS}(\mathrm{ESI})[\mathrm{M}+\mathrm{H}]^{+} 354.2$, $[\mathrm{M}+\mathrm{Na}]^{+} 376.2$.

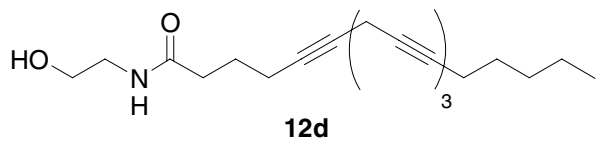

12d: resin $5 \mathbf{b}$ (43.3mg, $0.028 \mathrm{mmol})$ was cleaved by LDA (15 eq) and ethanolamine (20 eq) at $10^{\circ} \mathrm{C}$ and $18 \mathrm{~h}$ at $\mathrm{rt}$ to give $\mathbf{1 2 d}(6.8 \mathrm{mg}, 74 \%)$, purity $55 \%$. LC/MS (ESI) $[\mathrm{M}+\mathrm{H}]^{+} 340.2,[\mathrm{M}+\mathrm{Na}]^{+} 362.2$. 


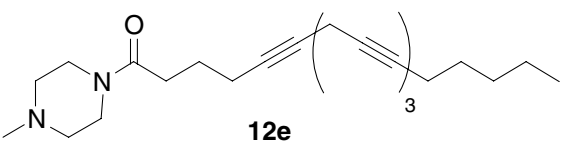

12e: resin $\mathbf{5 b}(105.0 \mathrm{mg}, 0.068 \mathrm{mmol})$ was cleaved by method B to give 12e $(17.0 \mathrm{mg}, 66 \%)$, purity $66 \% .{ }^{1} \mathrm{H} \mathrm{NMR}\left(\mathrm{CDCl}_{3}, 500 \mathrm{MHz}\right): \delta 3.49$ $(\mathrm{m}, 4 \mathrm{H}), 3.14(\mathrm{~m}, 6 \mathrm{H}), 2.84(\mathrm{~m}, 6 \mathrm{H}), 2.47(\mathrm{~m}, 4 \mathrm{H}), 2.15(\mathrm{~m}, 2 \mathrm{H}), 1.85(\mathrm{~m}, 2 \mathrm{H}), 1.56(\mathrm{~m}$, 6H) 0.89 (t, $J=7.0 \mathrm{~Hz}, 3 \mathrm{H})$. LC/MS (ESI) $[\mathrm{M}+\mathrm{H}]^{+} 379.2,[\mathrm{M}+\mathrm{Na}]^{+} 401.2$.

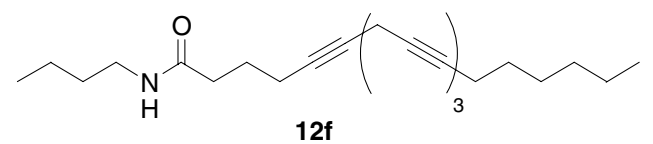

12f: resin 5d (153 mg, $0.16 \mathrm{mmol})$ was cleaved by method B to give 12f $(32.8 \mathrm{mg}, 88 \%)$, purity $40 \%$. LC/MS (ESI) $[\mathrm{M}+\mathrm{H}]^{+} 366.2$, $[\mathrm{M}+\mathrm{Na}]^{+}$388.2.

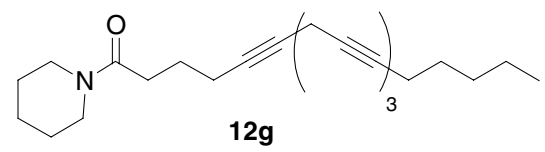

12g: resin 5b (114.7 $\mathrm{mg}, 0.068 \mathrm{mmol})$ was treated by method B to give $12 \mathrm{~g}(19.4 \mathrm{mg}, 79 \%)$ with purity $75 \%$. ${ }^{1} \mathrm{H} \mathrm{NMR}\left(\mathrm{CDCl}_{3}, 500 \mathrm{MHz}\right): \delta$ 3.55 (t, J=5.5 Hz, 2H), 3.43 (t, J=5.5 Hz, 2H), $3.14(\mathrm{~m}, 6 \mathrm{H}), 2.43$ (t, J=7.4 Hz, 2H) 2.25 (t, $J=6.9 \mathrm{~Hz}, 2 \mathrm{H}), 2.14(\mathrm{t}, J=7.2 \mathrm{~Hz}, 2 \mathrm{H}), 1.83(\mathrm{~m}, 2 \mathrm{H}), 1.58(\mathrm{~m}, 4 \mathrm{H}) 1.54(\mathrm{~m}, 6 \mathrm{H}), 1.32$ $(\mathrm{m}, 2 \mathrm{H}), 0.90(\mathrm{t}, J=7.00 \mathrm{~Hz}, 3 \mathrm{H})$. LC/MS (ESI) $[\mathrm{M}+\mathrm{H}]^{+} 364.2,[\mathrm{M}+\mathrm{Na}]^{+} 386.2$<smiles>CCC(C)(C)CC#CCCCC(=O)N1CCCCC1</smiles>

12h: resin 5a $(71.2 \mathrm{mg}, 0.031 \mathrm{mmol})$ was treated by method B to give 12h $(6.8 \mathrm{mg}, 65 \%)$, purity $83 \%$. ${ }^{1} \mathrm{H} \mathrm{NMR}\left(\mathrm{CDCl}_{3}, 400 \mathrm{MHz}\right): \delta 3.70(\mathrm{t}$, $J=6.3 \mathrm{~Hz}, 2 \mathrm{H}), 3.54(\mathrm{t}, J=5.5 \mathrm{~Hz}, 2 \mathrm{H}), 3.41(\mathrm{~m}, 2 \mathrm{H}), 3.13(\mathrm{~m}, 6 \mathrm{H}), 2.43(\mathrm{~m}, 2 \mathrm{H}), 2.24(\mathrm{~m}$, $2 \mathrm{H}), 1.87(\mathrm{~m}, 2 \mathrm{H}), 1.62(\mathrm{~m}, 2 \mathrm{H}), 1.54(\mathrm{~m}, 4 \mathrm{H}), 1.24(\mathrm{~m}, 2 \mathrm{H}) . \mathrm{LC} / \mathrm{MS}(\mathrm{ESI})[\mathrm{M}+\mathrm{H}]^{+}$ 338.2, $[\mathrm{M}+\mathrm{Na}]^{+} 360.1$.

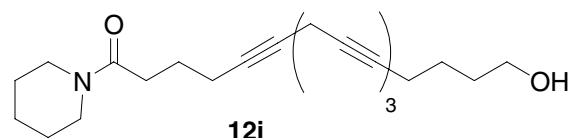

12i: resin 5c (97.2 $\mathrm{mg}, 0.0447 \mathrm{mmol})$ was treated by method B to give 12i $(6.1 \mathrm{mg}, 37 \%)$, purity $86 \% .{ }^{1} \mathrm{H} \mathrm{NMR}\left(\mathrm{CDCl}_{3}, 400 \mathrm{MHz}\right): \delta 3.67(\mathrm{t}$, $J=6.30 \mathrm{~Hz}, 2 \mathrm{H}), 3.54(\mathrm{t}, J=5.4 \mathrm{0Hz}, 2 \mathrm{H}), 3.42(\mathrm{~m}, 2 \mathrm{H}), 3.13(\mathrm{~m}, 6 \mathrm{H}), 2.43(\mathrm{~m}, 2 \mathrm{H}), 2.28$ $(\mathrm{m}, 2 \mathrm{H}), 1.84(\mathrm{~m}, 4 \mathrm{H}), 1.6-1.54(\mathrm{~m}, 10 \mathrm{H})$. LC/MS (ESI) $[\mathrm{M}+\mathrm{H}]^{+}$366.2, $[\mathrm{M}+\mathrm{Na}]^{+} 388.1$.

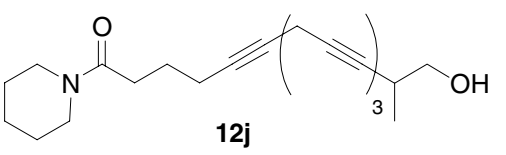

12j: resin 5e (137 mg, $0.17 \mathrm{mmol})$ was treated by method B to give 12j $(23.1 \mathrm{mg}, 40 \%)$, purity $20 \%$. LC/MS (ESI) $[\mathrm{M}+\mathrm{H}]^{+} 352.1,\left[\mathrm{M}+\mathrm{H}-\mathrm{H}_{2} \mathrm{O}\right]^{+}$ 334.1. 


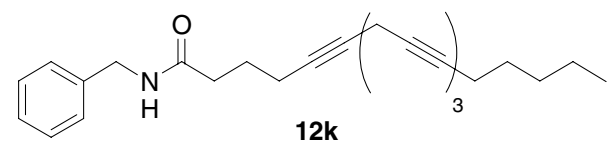

12k: resin $5 \mathbf{b}(82.9 \mathrm{mg}, 0.069 \mathrm{mmol})$ was treated by method B to give $12 \mathrm{k}(15.1 \mathrm{mg}, 57 \%)$ with purity $54 \% .{ }^{1} \mathrm{H} \mathrm{NMR}\left(\mathrm{CDCl}_{3}, 500 \mathrm{MHz}\right): \delta$ $7.34(\mathrm{~m}, 2 \mathrm{H}), 7.32(\mathrm{~m}, 3 \mathrm{H}), 6.99(\mathrm{~s}, 1 \mathrm{H}), 4.45(\mathrm{~m}, 2 \mathrm{H}), 3.11(\mathrm{~m}, 6 \mathrm{H}), 2.35(\mathrm{t}, J=7.70 \mathrm{~Hz}$, $2 \mathrm{H}), 2.24(\mathrm{~m}, 4 \mathrm{H}), 2.14(\mathrm{~m}, 2 \mathrm{H}), 1.85(\mathrm{~m}, 2 \mathrm{H}), 1.47(\mathrm{~m}, 2 \mathrm{H}), 1.32(\mathrm{~m}, 2 \mathrm{H}), 089(\mathrm{t}$, $J=7.02 \mathrm{~Hz}, 3 \mathrm{H})$. LC/MS (ESI) $[\mathrm{M}+\mathrm{H}]^{+} 386.1$.

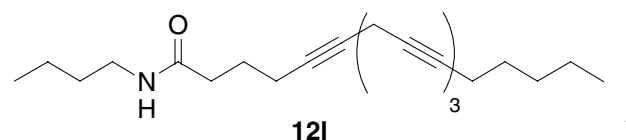

12l: resin $\mathbf{5 b}(97.3 \mathrm{mg}, 0.082 \mathrm{mmol})$ was treated by method B to provide $12 \mathrm{l}(15.2 \mathrm{mg}, 53 \%)$ with purity $70 \% .{ }^{1} \mathrm{H} \mathrm{NMR}\left(\mathrm{CDCl}_{3}, 500 \mathrm{MHz}\right)$ :

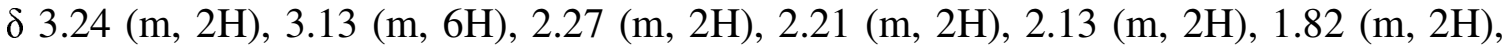
$1.74(\mathrm{~m}, 2 \mathrm{H}), 1.32(\mathrm{~m}, 4 \mathrm{H}), 0.89-0.91(\mathrm{~m}, 6 \mathrm{H}) . \mathrm{LC} / \mathrm{MS}(\mathrm{ESI})[\mathrm{M}+\mathrm{H}]^{+}$352.2, $[\mathrm{M}+\mathrm{Na}]^{+}$ 374.2 .

Compounds 4a-l were prepared from 12a-l by $\mathrm{P}-2 \mathrm{Ni}$ catalytic hydrogenation $\left(\mathrm{Ni}(\mathrm{OAc})_{2} \cdot 4 \mathrm{H}_{2} \mathrm{O} 2.24 \mathrm{eq} ; \mathrm{NaBH}_{4} 2.24 \mathrm{eq}, 1 \mathrm{M}\right.$ in absolute EtOH; ethylene diamine 2.24 eq; $\mathrm{H}_{2}$ balloon; $3-4 \mathrm{~h}$; rt) and the products were purified by PTLC.

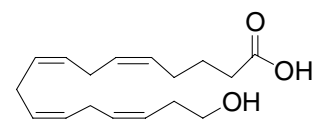

4a $40 \% \quad 4 a$ : by the general method, the reduction of $12 \mathrm{a}$ gave $4 \mathrm{a}$ in $40 \%$ yield. ${ }^{1} \mathrm{H} \mathrm{NMR}\left(\mathrm{CDCl}_{3}, 400 \mathrm{MHz}\right): \delta 5.3-5.5(\mathrm{~m}, 8 \mathrm{H}), 3.70(\mathrm{t}, J=6.545 \mathrm{~Hz}, 2 \mathrm{H}), 2.85(\mathrm{~m}, 6 \mathrm{H})$, $2.40(\mathrm{~m}, 5 \mathrm{H}), 2.15(\mathrm{~m}, 2 \mathrm{H}), 2.05(\mathrm{~m}, 2 \mathrm{H}), 1.70(\mathrm{t}, J=7.3 \mathrm{~Hz}, 3 \mathrm{H}), 0.80(\mathrm{~m}, 3 \mathrm{H})$. HRMS (MALDI-FTMS) calcd. for $\mathrm{C}_{17} \mathrm{H}_{26} \mathrm{O}_{3}[\mathrm{M}+\mathrm{Na}]^{+} 301.1774$, found: 301.1766 .

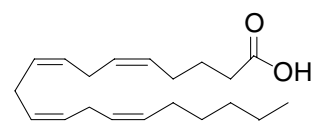

4b $34 \% \quad 4 b$ : yield 34\%. ${ }^{1} \mathrm{H}$ NMR $\left(\mathrm{CDCl}_{3}, 500 \mathrm{MHz}\right): \delta 5.40(\mathrm{~m}, 8 \mathrm{H}), 2.80(\mathrm{~m}$, $6 \mathrm{H}), 2.40(\mathrm{~m}, 2 \mathrm{H}), 2.15(\mathrm{~m}, 2 \mathrm{H}), 2.05(\mathrm{~m}, 2 \mathrm{H}), 1.70(\mathrm{~m}, 2 \mathrm{H}), 1.30(\mathrm{~m}, 2 \mathrm{H}), 0.85(\mathrm{~m}, 3 \mathrm{H})$. ${ }^{13} \mathrm{C}$ NMR $\left(500 \mathrm{MHz}, \mathrm{CDCl}_{3}\right): \delta 130.51,129.04,128.74,128.56,127.85,127.52,63.07$, $32.79,31.51,29.70,27.20,26.43,25.60,24.50,22.57,14.07 . \mathrm{MS}$ (ESI) [M-H] 303.

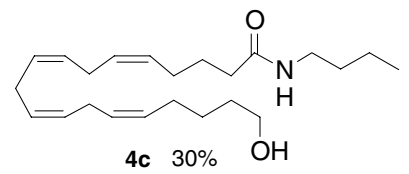

$(\mathrm{t}, J=6.6 \mathrm{~Hz}, 2 \mathrm{H}), 3.25(\mathrm{q}, J=6.5 \mathrm{~Hz}, 2 \mathrm{H}), 2.80(\mathrm{~m}, 6 \mathrm{H}), 2.10(\mathrm{~m}, 4 \mathrm{H}), 1.70(\mathrm{~m}, 4 \mathrm{H}), 1.60$ $(\mathrm{m}, 4 \mathrm{H}), 1.50(\mathrm{~m}, 2 \mathrm{H}), 1.40(\mathrm{~m}, 2 \mathrm{H}), 0.90(\mathrm{t}, J=7.3 \mathrm{~Hz}, 3 \mathrm{H}) .{ }^{13} \mathrm{C}$ NMR $(500 \mathrm{MHz}$, $\left.\mathrm{CDCl}_{3}\right): \delta 172.78,129.91,129.15,129.08,128.78,128.71,128.38,128.15,128.00,68.13$, 62.78, 39.21, 36.15, 32.36, 31.72, 30.94, 26.96, 26.67, 25.72, 25.65, 25.56, 20.06, 13.75 . LC/MS (ESI) $[\mathrm{M}+\mathrm{H}]^{+} 362,[\mathrm{M}+\mathrm{Na}]^{+} 384$. 


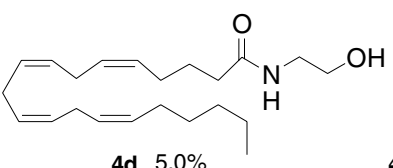

4d $5.0 \%$

4d: yield 5.0\%. ${ }^{1} \mathrm{H}$ NMR $\left(\mathrm{CDCl}_{3}, 400 \mathrm{MHz}\right): \delta 5.40(\mathrm{~m}, 8 \mathrm{H})$,

$3.75(\mathrm{t}, J=4.7 \mathrm{~Hz}, 2 \mathrm{H}), 3.45(\mathrm{q}, J=5.2 \mathrm{~Hz}, 2 \mathrm{H}), 2.85(\mathrm{~m}, 6 \mathrm{H}), 2.35(\mathrm{t}, J=7.5 \mathrm{~Hz}, 2 \mathrm{H}), 2.25$ $(\mathrm{q}, J=6.5 \mathrm{~Hz}, 2 \mathrm{H}), 2.10(\mathrm{q}, J=6.4 \mathrm{~Hz}, 2 \mathrm{H}), 1.30(\mathrm{~m}, 8 \mathrm{H}), 0.85(\mathrm{t}, J=6.5 \mathrm{~Hz}, 3 \mathrm{H}) .{ }^{13} \mathrm{C} \mathrm{NMR}$ $\left(500 \mathrm{MHz}, \mathrm{CDCl}_{3}\right): \delta 174.18,130.55,129.04,128.86,128.56,128.26,128.15,127.85$, 127.51, 62.75, 42.49, 35.92, 31.52, 29.71, 29.33, 27.23, 26.62, 25.65, 25.43, 22.59, 14.09. HRMS (MALDI-FTMS) calcd. for $\mathrm{C}_{22} \mathrm{H}_{37} \mathrm{NO}_{2}[\mathrm{M}+\mathrm{Na}]^{+} 370.2716,370.2720$.

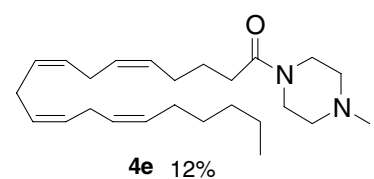

4e: yield $12 \% .{ }^{1} \mathrm{H}$ NMR $\left(\mathrm{CDCl}_{3}, 500 \mathrm{MHz}\right): \delta 5.40(\mathrm{~m}, 8 \mathrm{H}), 4.20$ $(\mathrm{m}, 3 \mathrm{H}), 3.60(\mathrm{~m}, 4 \mathrm{H}), 3.45(\mathrm{~m}, 4 \mathrm{H}), 2.80(\mathrm{~m}, 6 \mathrm{H}), 2.40(\mathrm{~m}, 4 \mathrm{H}), 2.30(\mathrm{~m}, 2 \mathrm{H}), 1.60(\mathrm{~m}$, $2 \mathrm{H}), 1.40(\mathrm{~m}, 4 \mathrm{H}), 0.90(\mathrm{~m}, 3 \mathrm{H})$. HRMS (MALDI-FTMS) calcd. for $\mathrm{C}_{25} \mathrm{H}_{42} \mathrm{~N}_{2} \mathrm{O}$ $[\mathrm{M}+\mathrm{Na}]^{+}$409.3189, found 409.3171. LC/MS (ESI) $[\mathrm{M}+\mathrm{H}]^{+}$387.3, $[\mathrm{M}+\mathrm{Na}]^{+}$409.3.

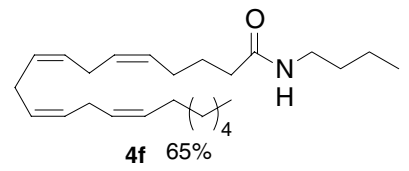
(m, $2 \mathrm{H}), 2.90(\mathrm{~m}, 6 \mathrm{H}), 2.80(\mathrm{~m}, 2 \mathrm{H}), 2.27(\mathrm{~m}, 2 \mathrm{H}), 2.20(\mathrm{~m}, 2 \mathrm{H}), 1.70(\mathrm{~m}, 2 \mathrm{H}), 1.50(\mathrm{~m}$, $4 \mathrm{H}), 1.30(\mathrm{~m}, 8 \mathrm{H}), 0.92(\mathrm{t}, J=7.2 \mathrm{~Hz}, 3 \mathrm{H}) 0.88(\mathrm{~m}, 3 \mathrm{H})$. LC/MS (ESI) $[\mathrm{M}+\mathrm{H}]^{+} 374.3$, $[\mathrm{M}+\mathrm{Na}]^{+}$396.3. HRMS (MALDI-FTMS) calcd. for $\mathrm{C}_{25} \mathrm{H}_{43} \mathrm{NO}[\mathrm{M}+\mathrm{Na}]^{+} 396.3237$, found 396.3235 .

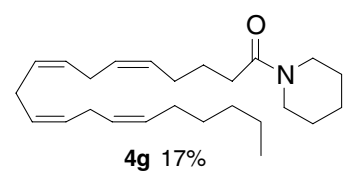
=5.4 Hz, 2H), $3.35(\mathrm{t}, 5.3 \mathrm{~Hz}, 2 \mathrm{H}), 2.89(\mathrm{~m}, 6 \mathrm{H}), 2.30(\mathrm{~m}, 2 \mathrm{H}), 2.00-2.15(\mathrm{~m}, 4 \mathrm{H}), 1.60$ $(\mathrm{m}, 6 \mathrm{H}), 1.20-1.30(\mathrm{~m}, 8 \mathrm{H}), 0.90(\mathrm{t}, J=6.7 \mathrm{~Hz}, 3 \mathrm{H})$. HRMS (MALDI-FTMS) calcd. for $\mathrm{C}_{25} \mathrm{H}_{41} \mathrm{NO}[\mathrm{M}+\mathrm{H}]^{+} 372.3261$, found 372.3265. LC/MS (ESI) $[\mathrm{M}+\mathrm{H}]^{+} 372.3,[\mathrm{M}+\mathrm{Na}]^{+}$ 394.3.

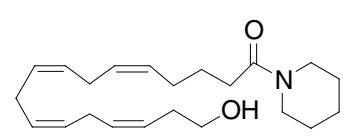

4h $18 \% \quad$ 4h: yield $18 \% .{ }^{1} \mathrm{H}$ NMR $\left(\mathrm{CDCl}_{3}, 500 \mathrm{MHz}\right): \delta 5.38(\mathrm{~m}, 8 \mathrm{H}), 3.75(\mathrm{t}$, $J=6.5 \mathrm{~Hz}, 2 \mathrm{H}), 3.55(\mathrm{t}, J=5.5 \mathrm{~Hz}, 2 \mathrm{H}), 3.35(\mathrm{~m}, 2 \mathrm{H}), 3.35(\mathrm{~m}, 6 \mathrm{H}), 2.35(\mathrm{~m}, 2 \mathrm{H}), 2.20(\mathrm{~m}$, $2 \mathrm{H}), 2.00(\mathrm{~m}, 2 \mathrm{H}), 1.60(\mathrm{~m}, 6 \mathrm{H}), 1.40(\mathrm{~m}, 2 \mathrm{H})$. HRMS (MALDI-FTMS) calcd. for $\mathrm{C}_{22} \mathrm{H}_{35} \mathrm{NO}_{2}[\mathrm{M}+\mathrm{Na}]^{+}$368.256, found 368.2555. LC/MS [M+H] ${ }^{+}$346.2, $[\mathrm{M}+\mathrm{Na}]^{+}$368.2.

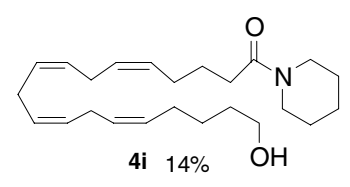

4i: yield 14\%. LC/MS $[\mathrm{M}+\mathrm{H}]^{+}$374.2, $[\mathrm{M}+\mathrm{Na}]^{+} 396.3$. 


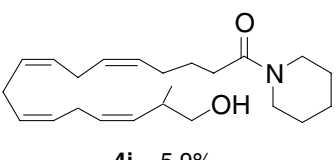

4j $5.9 \% \quad 4 j$ : yield $5.9 \%$. ${ }^{1} \mathrm{H}$ NMR $\left(\mathrm{CDCl}_{3}, 500 \mathrm{MHz}\right): \delta 5.40(\mathrm{~m}, 8 \mathrm{H}), 3.55$ (br, 2H), 3.40 (br, 2H), 3.20 (m, 2H), 2.84 (m, 6H), 2.33 (m, 3H), 2.15 (m, 2H), 1.50-1.70 $(\mathrm{m}, 6 \mathrm{H}), 1.30(\mathrm{~m}, 3 \mathrm{H}) .{ }^{13} \mathrm{C} \mathrm{NMR}\left(500 \mathrm{MHz}, \mathrm{CDCl}_{3}\right): \delta 199.54,130.96,129.43,128.87$, 128.70, 128.36, 68.14, 38.69, 30.33, 29.69, 28.91, 24.59, 23.72, 22.98, 14.05, 10.95 . HRMS (MALDI-FTMS) calcd. for $\mathrm{C}_{23} \mathrm{H}_{37} \mathrm{NO}_{2}[\mathrm{M}+\mathrm{Na}]^{+}$382.2716, found 382.2723.

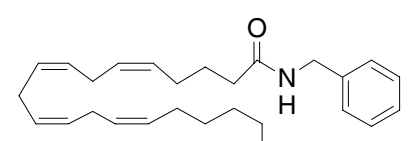

4k $21 \%$ 4k: yield 21\%. ${ }^{1} \mathrm{H} \mathrm{NMR}\left(\mathrm{CDCl}_{3}, 400 \mathrm{MHz}\right): \delta 7.20-7.40(\mathrm{~m}, 5 \mathrm{H})$, $5.35(\mathrm{~m}, 8 \mathrm{H}), 4.45(\mathrm{~d}, J=5.6 \mathrm{~Hz}, 2 \mathrm{H}), 2.79(\mathrm{~m}, 6 \mathrm{H}), 2.22(\mathrm{~m}, 2 \mathrm{H}), 2.00(\mathrm{~m}, 4 \mathrm{H}), 1.75(\mathrm{~m}$, $4 \mathrm{H}), 1.30(\mathrm{~m}, 4 \mathrm{H}), 0.90(\mathrm{t}, J=6.0 \mathrm{~Hz}, 3 \mathrm{H}) .{ }^{13} \mathrm{C} \mathrm{NMR}\left(500 \mathrm{MHz}, \mathrm{CDCl}_{3}\right): \delta 172.60$, $138.28,130.51,129.06,128.78,128.72,128.56,128.20,128.13,127.85,127.53,127.49$, 43.62, 36.07, 31.49, 29.69, 29.29, 27.19, 27.07, 26.66, 25.61, 25.49, 22.56, 14.07. HRMS (MALDI-FTMS) calcd. for $\mathrm{C}_{27} \mathrm{H}_{39} \mathrm{NO}[\mathrm{M}+\mathrm{Na}]^{+}$416.2924, found 416.2927. ESI [M+Na] ${ }^{+}$ 416, $[\mathrm{M}+\mathrm{Cl}]^{-}$428. LC/MS $[\mathrm{M}+\mathrm{H}]^{+}$394.2, $[\mathrm{M}+\mathrm{Na}]^{+} 416.2$.

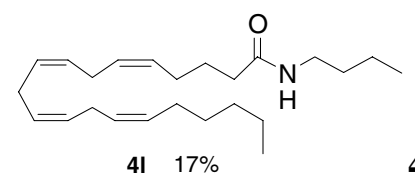

$(\mathrm{q}, J=6.7 \mathrm{~Hz}, 2 \mathrm{H}), 2.70-2.90(\mathrm{~m}, 6 \mathrm{H}), 2.20(\mathrm{~m}, 6 \mathrm{H}), 1.80(\mathrm{~m}, 2 \mathrm{H}), 1.70(\mathrm{~m}, 2 \mathrm{H}), 1.50(\mathrm{~m}$ $4 \mathrm{H}), 1.30(\mathrm{~m}, 4 \mathrm{H}), 0.90(\mathrm{~m}, 6 \mathrm{H}) .{ }^{13} \mathrm{C} \mathrm{NMR}\left(500 \mathrm{MHz}, \mathrm{CDCl}_{3}\right): \delta 187.10,128.93,128.79$, $128.18,127.85,127.62,127.27,125.27,124.29$, 66.96, 39.24, 36.21, 31.75, 27.23, 25.65, 24.76, 22.59, 22.24, 20.09, 14.09, 13.77. HRMS (MALDI-FTMS) calcd. for $\mathrm{C}_{24} \mathrm{H}_{41} \mathrm{NO}$ $[\mathrm{M}+\mathrm{H}]^{+}$360.3261, found 360.3256. LC/MS $[\mathrm{M}+\mathrm{H}]^{+} 360.3,[\mathrm{M}+\mathrm{Na}]^{+} 382.3$.

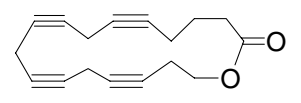

13a 13a: ${ }^{1} \mathrm{H} \mathrm{NMR}\left(\mathrm{CDCl}_{3}, 500 \mathrm{MHz}\right): \delta 4.42(\mathrm{t}, J=6.9 \mathrm{~Hz}, 2 \mathrm{H}), 3.14(\mathrm{~m}$, $6 \mathrm{H}), 2.64$ (tt, $J=6.9 \mathrm{~Hz}, 2.26 \mathrm{~Hz}, 2 \mathrm{H}), 2.49$ (t, $J=7.4 \mathrm{~Hz}, 2 \mathrm{H}), 2.27$ (tt, J=6.9 Hz, $2.3 \mathrm{~Hz}$, 2H), 1.83 (quint, $J=7.1 \mathrm{~Hz}, 2 \mathrm{H}) .{ }^{13} \mathrm{C} \mathrm{NMR}\left(500 \mathrm{MHz}, \mathrm{CDCl}_{3}\right): \delta 177.86,79.31,76.34$, 75.02, 74.88, 74.58, 74.52, 74.29, 74.10, 65.53, 32.44, 29.69, 23.49, 18.88, 18.05, 9.75. LC/MS ESI $[\mathrm{M}+\mathrm{H}]^{+} 251.3$ 